\title{
Gerechte Informationsgesellschaft: eine Frage politischen Willens
}

\section{Chantal Peyer}

\section{(2) OpenEdition \\ Journals}

Electronic version

URL: http://journals.openedition.org/sjep/549

DOI: $10.4000 /$ sjep.549

ISSN: 1663-9677

\section{Publisher}

Institut de hautes études internationales et du développement

\section{Printed version}

Date of publication: 1 novembre 2003

Number of pages: 135-137

ISSN: 1660-5926

\section{Electronic reference}

Chantal Peyer, "Gerechte Informationsgesellschaft: eine Frage politischen Willens », Schweizerisches Jahrbuch für Entwicklungspolitik [Online], 22-2 | 2003, Online erschienen am: 10 Juni 2010, abgerufen am 08 September 2020. URL : http://journals.openedition.org/sjep/549; DOI : https://doi.org/ 10.4000/sjep.549 


\title{
Gerechte Informationsgesellschaft: eine Frage politischen Willens
}

\author{
Chantal Peyer*
}

W ir lassen uns von der technizistischen, neoliberalen und von Illusionen des Egalitarismus geprägten Ideologie des ,Global Village' nicht täuschen: Wegen des technologischen Grabens und der ungleich verteilten Kenntnisse bedeutet das Internet nicht Wissen für alle, sondern es macht einmal mehr eine Welt der zwei Geschwindigkeiten deutlich.“ Dies schrieb Olivier Barlet im Jahr 2000 in einem Leitartikel zu Africultures $^{1}$. Wie steht es damit zwei Jahre später, im Vorfeld des Weltgipfels über die Informationsgesellschaft (WSIS)? Wie werden das Potenzial und die Gefahren der Informationsgesellschaft wahrgenommen?

„Brot für alle“ ist der Ansicht, dass die neuen Informations- und Kommunikationstechnologien (NIKT) eine Chance sein können. Mit den drahtlosen Technologien zum Beispiel - allgemein bekannt unter dem Kürzel WiFi - kann man die Investition in teure und schnell veraltete Infrastrukturen limitieren ${ }^{2}$. Die Entwicklung der Kommunikationsmittel erleichtert die Schaffung von Datenbanknetzwerken und kann den Einbezug der Bevölkerung stärken. Ihre Anwendungen in Fernmedizin und
Fernunterricht oder die Schaffung von Zentren mit kollektivem Zugang sind wichtige Instrumente im Kampf gegen die Armut.

Aber die NIKT sind nicht Zweck, sondern Mittel. Ohne klaren politischen Willen, ohne einen Paradigmenwechsel ist das Erreichen eines gerechten „globalen Dorfes“ eine Illusion. Heute nutzen nur 5\% der Weltbevölkerung das Internet, und von diesen leben $88 \%$ in den Ländern des Nordens. Im Süden wird das Internet vor allem von Männern aus städtischen Gebieten und mit guter Ausbildung genutzt. So wie sich die Dinge heute entwickeln, vergrössert die Informationsgesellschaft demnach die wirtschaftlichen, sozialen und kulturellen Gräben.

Wird sich der politische Wille am Weltgipfel über die Informationsgesellschaft einstellen? Die Resultate der ersten Vorbereitungskonferenzen lassen Zweifel aufkommen. Für Brot für alle müsste die in der ersten Phase des Gipfels in Genf verabschiedete Erklärung im Grundsatz auf den bestehenden rechtlichen Rahmen aufbauen, namentlich auf der Allgemeinen Erklärung der Menschenrechte (1948). Artikel 19 dieser Erklärung

* Programmbeauftragte bei Brot für alle und Mitglied der Schweizer Delegation am Weltgipfel über die Informationsgesellschaft, Schweiz.

Africultures, Internet en Afrique, no 23, Dezember 1999 <www.africultures.com>.

Alan Levy, Matching New WiFi Technology With Virtual Private Networks To Create Affordable Universal Internet Access <www.bytesforall.org/index_connectivity_top1.htm>. 
hält fest, dass ,jeder Mensch das Recht auf Meinungsfreiheit und auf freie Meinungsäusserung“ hat. „Dieses Recht schliesst die Freiheit ein, Meinungen ungehindert äussern sowie die Freiheit in Medien - jeder Art und ohne Rücksicht auf Grenzen Informationen und Gedankengut $\mathrm{zu}$ suchen, zu empfangen und zu verbreiten“. Das entspricht auch den wesentlichen Forderungen der Zivilgesellschaft: Zugang aller zu den Kommunikationsmitteln (Infrastrukturen) sowie zum Empfang und zur Erzeugung von Information (Inhalte). Zwar sind sich die Regierungen in der Anerkennung dieser Prinzipien mehr oder weniger einig; die Umsetzung stockt jedoch aus mindestens drei Gründen :

Erstens muss für eine Respektierung des Rechts auf Kommunikation der Mensch ins Zentrum gestellt werden. Dies ruft die Schweizer Plattform für die Informationsgesellschaft - ein Verband von Nichtregierungsorganisationen und Medienleuten, die sich am WSIS-Prozess beteiligen - seit ihrer Gründung 2002 immer wieder in Erinnerung. Im WSIS-Prozess jedoch wird ,der Schwerpunkt mehr auf die Infrastrukturen (für den Süden) und die potenziellen Märkte (für den Norden) als auf die wirklichen Rechte und Bedürfnisse der Menschen und der Gemeinschaften gelegt" ${ }^{63}$.

Zweitens bedingt der Aufbau einer demokratischen und partizipativen Informationsgesellschaft eine Grundsatzdebatte über Fragen wie Rechte des geistigen Eigentums, Respektie- rung der Menschenrechte und der freien Meinungsäusserung sowie Medienkonzentration. All diese Themen stehen im Zentrum der Anliegen der Zivilgesellschaft ${ }^{4}$. In den offiziellen Verhandlungen des Gipfels nehmen sie aber jetzt schon lediglich einen kleinen Platz ein oder sind gar ausgeschlossen.

Und schliesslich fehlt jede konkrete Verpflichtung: Um den digitalen Graben zu überwinden und den Zugang zu Mitteln und Inhalten der Information für alle $\mathrm{zu}$ ermöglichen, sind innovative öffentliche Politiken und bedeutende Finanzen nötig. Aber der Vorschlag zur Schaffung eines internationalen Solidaritätsfonds für die Informationsgesellschaft und die Unterstützung für freie Software um nur zwei Beispiele zu nennen stossen im Rahmen des WSIS kaum auf Echo. Die Empfehlungen, die den Entwicklungsländern zur Überwindung des digitalen Grabens abgegeben werden, gehen alle in die gleiche Richtung: Den Telekommarkt privatisieren, stabile und attraktive gesetzliche Rahmenbedingungen für die Privatunternehmen einführen und sich an öffentlich-privaten Partnerschaften beteiligen. Doch die Grenzen der gegenwärtigen Privatisierungs- und Liberalisierungspolitiken unter der Ägide der Welthandelsorganisation und der Internationalen Fernmeldeunion (UIT) sind bekannt: Sie erlauben es nicht, für die benachteiligten Regionen und Bevölkerungen der Entwicklungsländer angemessene Infrastrukturen $\mathrm{zu}$ erschwinglichen Preisen zu entwickeln ${ }^{5}$.

\footnotetext{
Website der Schweizer Plattform zur Informationsgesellschaft : <www.comunica-ch.net.>.

Vgl. z.B. die Kampagne CRIS (Communication Rights in the Information society) <www.crisinfo.org $>$. Vgl. dazu die Artikel von Jean-Louis Fullsack, namentlich, L'UIT, la vieille dame des télécommunications dans la tourmente libérale <www.globenet.org/csdptt.> und die Artikel von Annie ChéneauLoquay: <www.africanti.org $>$.
} 
Zum Aufbau einer wirklichen Partnerschaft, und um Lösungen für die Probleme der Informationsgesellschaft $\mathrm{zu}$ finden, müssen deshalb dringend neue Formen der Zusammenarbeit, der Beratung oder gar der Regierungstätigkeit gefunden werden. Mehr als in jedem anderen Bereich besitzen die Zivilgesellschaft und der Privatsektor ein Know-how und eine Kreativität, welche beim künftigen Aufbau der Informationsgesellschaft einbezogen werden müssen. Deshalb hat die Schweizer Plattform zur Schaffung einer hochrangigen schweizerischen Task-Force aufgerufen, in der all diese Akteure vertreten sein sollten, welche einen Beitrag zum Prozess des Gipfels leisten können (Verantwortliche der zuständigen Ämter, Parlamentsmitglieder, Delegierte des Privatsektors, der Zivilgesellschaft und der Medien). Eine solche wirklich tripartite, ständige und transparente Task-Force wäre der Ort, wo namentlich die Positionen und Vorschläge der Schweiz für den Gipfel ausgehandelt und erarbeitet werden sollten. Wenn die Schweizer Regierung diesen Vorschlag annimmt, könnte sie damit ein Beispiel geben, und die offiziellen Texte des Gipfels, welche die Ausarbeitung neuer tripartiter Beratungsformen verlangen, wortwörtlich befolgen ${ }^{6}$.

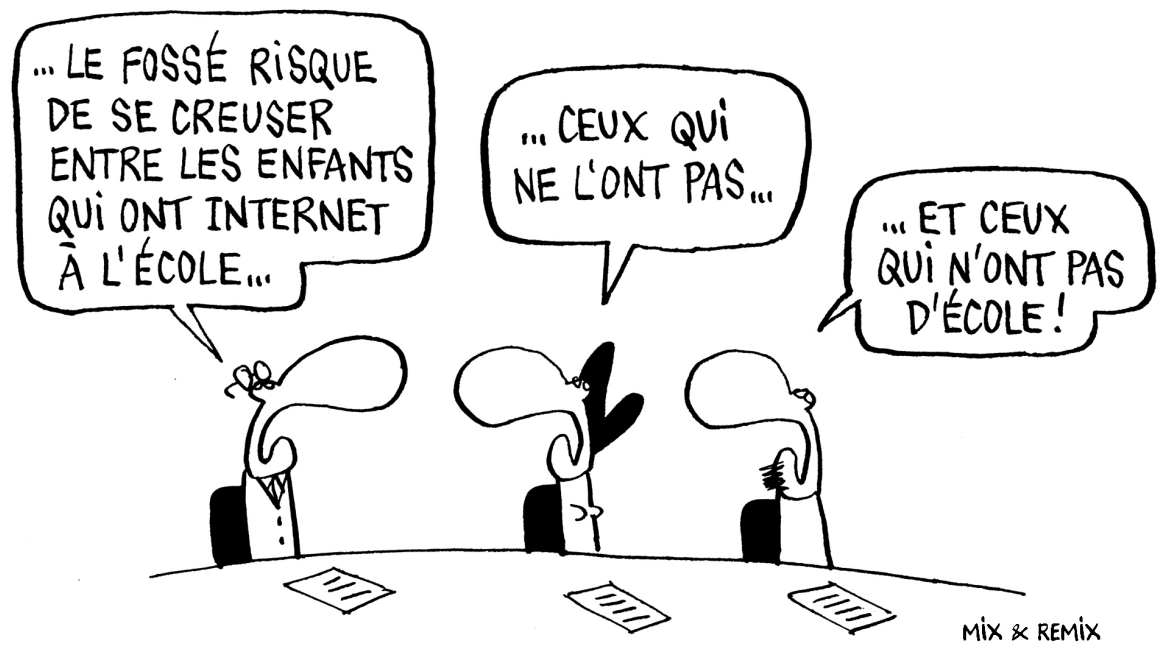

Die digitale Kluft wird immer tiefer zwischen Kindern, die in der Schule Internet haben... Kindern ohne Internet in der Schule... und Kindern ohne Schule!

6 Vgl. zum Beispiel die Resolution 56/183 der UNO-Generalversammlung, welche ,nichtstaatliche Organisationen und den Privatsektor“ ermutigt, ,zu dem zwischenstaatlichen Vorbereitungsprozess und zu dem Gipfel selbst Beiträge zu leisten und aktiv daran mitzuwirken“. 